\section{Lung cancer patient needs in different countries}

\author{
“...there is a strong need to overcome many challenges and \\ barriers to ensure that all of the patients may have the same hope \\ and equal chances to fight against this disease regardless of the \\ country of residence."
}

\begin{abstract}
Stefania Vallone ${ }^{2}$, Ingeborg Beunders ${ }^{3}$ \& Ewelina Szmytke ${ }^{1,2}$
\end{abstract}
First draft submitted: 16 February 2017; Accepted for publication: 20 February 2017; Published online: 14 July 2017

Despite the advances that have been made in the last decades, lung cancer still represents one of the global health problems worldwide and most lung cancer patients have to deal with a number of unmet needs for which they require assistance. Lung cancer is considered an 'invisible' disease because of getting a low level of public attention. Regardless of its prevalence and the fact that it is one of the hardest cancers to diagnose and to treat, with a poor survival rate, it continues to be one of the most overlooked cancer type [1] and this sentiment is prevalent on a global scale. The poor outcomes of lung cancer and the persistent misconception that the majority of patients are smokers often generate a general negative perception and attitude toward this condition and a poor media coverage.

The challenges and the needs in this area are multiple and for most lung cancer patients the assessment is a critical but important step in order to receive high quality of care. With an estimated 1.8 million new cases [2] and 1.59 million deaths per year, lung cancer is one of the most common cancers worldwide and the leading cause of cancer-related deaths in the EU with 267,700 deaths recorded in 2012 and representing approximately 20\% of all cancer deaths [3].

Despite the huge health, social and economic burden associated with lung cancer, it falls far behind other cancers in terms of research, patient support and timely access to innovative therapies. These disparities have a profound impact on patient's quality of life and overall survival.

It is important to identify the patient priorities efficiently so that patients can be helped with appropriate resources.

Time is crucial for lung cancer patients. The common goal for them in any part of the Earth is to live longer, with a better quality of life. The treatment effectiveness and survival may be increased through early detection, accurate diagnosis and a fast and equal access to innovative treatments.

With the aim to investigate the unmet needs of people affected by lung cancer in different geographic areas, an anonymous survey was designed and implemented online from September to November 2016 through a global network of Lung Cancer Patient Advocacy Groups. The survey not only aimed on identifying the global challenges that lung cancer patients and their families have to face but also on gaining patients' ideas about measures that may improve their condition. The survey was filled in by 161 people across 21 countries and the results revealed that the three big issues are: late diagnosis, lack of research funding and stigma.

'Association For The Fight Against Lung Cancer in Poland, Gdansk, Poland

${ }^{2}$ Lung Cancer Europe (LuCE), Bern, Switzerland

${ }^{3}$ MAS, MBA, Focus patient Ltd, Baden, Austria

*Author for correspondence: Tel.: +39 0119026 980; stefania.vallone@womenagainstlungcancer.eu



\section{KEYWORDS}

- lung cancer • lung cancer challenges $\bullet$ patients' needs - stigma 
In addition, other needs were highlighted, such as the high costs of new cancer drugs, the delay or lack of access to innovative treatments, the lack of reliable information for patients and the lack of psychological help.

These results were discussed during the interactive Patient Advocacy Session held in Wien at World Conference on Lung Cancer (WCLC) 2016. The attendees from different parts of the world were asked to share their considerations about the main lung cancer patient needs in their countries. Data collected during the meeting, partly in accordance with the information from the previous survey, pointed out the fact that although the scenario of lung cancer improved in the last 10 years, there are still important issues that have to be faced. In every country there are influencing factors for patient's condition and needs, such as the healthcare system, the socio-economic context and the level of public awareness. Unfortunately, the number of people involved in this study is not representative for the entire lung cancer patient community in those countries, so the data just provide informational hints.

\section{Early diagnosis}

Late diagnosis remains the key challenge confirmed by both, the results of the survey and by the discussion with attendees coming from the USA, South America and Europe. The most effective treatment for lung cancer is a surgery [4] but it requires an early diagnosis. In such stage, the cancer would be more likely to be treated successfully and generally would increase the chances of survival and improve the quality of life of the patients and the caregivers.

The causes of late diagnosis are different:

- The low awareness of lung cancer signs and symptoms that causes people to see the GP rather late;

- Some people delay a check-up because they are worried about possible findings;

- Patients often are referred late to specialists for tests or treatments;

- Delays can also occur in getting an appointment at the hospital.

So on one hand, efforts should be made to reduce the time between primary care consultation and the beginning of the treatment [5]. On the other hand, action must be taken to improve awareness and knowledge about lung cancer symptoms among people, helping to recognize symptoms in early stages.

\section{Stigma}

The results of the survey confirmed that among the others in 2016, stigma is still one of the main perceived challenges in Europe, especially in Italy, Romania, The Netherlands, UK, Mexico, the USA and Australia.

Although it cannot be denied that smoking is the most important risk factor for developing lung cancer, misinformation or false myths generate fear and a negative perception of a person affected by lung cancer and create a noteworthy stigma due to the disease's link to smoking. Stigma persists, in part, because lung cancer is considered a self-inflicted disease and patients feel stigmatized for it, whether they smoked or not. A lot of people assume that smoking causes lung cancer, despite the fact that about $15 \%$ of lung cancer patients never smoked. The lack of public empathy and support adds an emotional burden to an already frustrating situation that can affect patient's quality of life. In 2010, a research carried out by Ipsos MORI [6] on behalf of the Global Lung Cancer Coalition, which surveyed over 16,000 people in 16 countries, found some evidence that sympathy levels were influenced by rates of smoking in each country [7]. Between 10 and 29\% of people admitted to feeling less sympathetic toward lung cancer sufferers because of its association with smoking. Generally people in countries with lower rates of smoking had a greater tendency to admit that they felt less sympathetic to people with lung cancer compared with other types of cancer. However, the pattern is not perfect, which suggests that other cultural or traditional factors may play an important role.

\section{Research}

In this disease area, it is essential to make improvements in prevention, diagnosis, treatments and support.

Having in mind the global impact of lung cancer, there are concerns about the adequacy of efforts and progress made in lung cancer research. To assess the situation, the Global Lung Cancer Coalition commissioned the Institute of Cancer Policy to examine the state of global lung cancer research in order to better understand what type of research is being carried out in lung 
cancer and to highlight which areas need additional investments. The study revealed that 24 countries [7] are responsible for approximately $97 \%$ of all lung cancer research articles published between 2004 and 2013 and although the number of papers published worldwide on this cancer has more than doubled from 2157 papers in 2004 to 4845 in 2013, the increase of the proportion of global research in lung cancer compared with other cancer types is minimal (4.4\% in 2004, 5.6\% in 2013). Apart from China, the research in most of other countries declined, particularly in Belgium, Poland, France, Norway, Italy, Spain, Greece and the Republic of Korea [8]. The majority of the research is dedicated to medicines, genetics and biomarkers, with less than $1 \%$ of it being dedicated to understand and improve palliative and support care and quality of life, despite the fact that many patients would benefit from early palliative care, given the poor survival rates with lung cancer [8]. More research is needed for a disease that stands for poor chances of survival.

Screening programs may represent a promising research field. In 2011, the National Lung Screening Trial in the USA demonstrated that screening with the use of low-dose computed tomography reduces mortality in lung cancer by $20 \%$, but while guidelines are common practice in the USA, more research is needed in Europe to gather data about the efficiency and the cost-effectiveness [9].

\section{Access to molecular testing \& innovative treatments}

In recent decades research has developed significant advancements in the landscape of lung cancer from chemotherapy through targeted therapies to the more recent immuno-oncology agents that have changed the approach to treat this disease and provided new therapeutic options in order to offer prolonged survival with fewer side effects and better quality of life. Knowledge of tumor molecular diversity has opened a new scenario for lung cancer treatments [10], and molecular testing is becoming a crucial part of the clinical decision process. Patients need to have an equal access to molecular testing and innovative drugs, however, lung cancer treatments widely vary and persisting inequalities in access have been registered in Europe, including differences in protocols for diagnosis and treatment, market access delays, high prices of drugs, regionalization of health systems and differences in reimbursement of innovative therapies. Furthermore, each country has its own centers of excellence. However, various factors (funding, resource and access) prevent the replication of this excellence throughout different countries and in many cases the best practice of one center is not shared with neighboring centers. But at the same time, high prices of these drugs currently represent one of the main global challenges and a major issue on the global political agenda. Considering that not all healthcare systems have been prepared for affording the costs and are now unable to reimburse these treatments, there are unacceptable disparities concerning access to medicines. Due to limited economic resources, there is a need of respecting budget constraints and consequently decisions on budget allocation become extremely difficult to take [11].

\section{Psychological help}

Around $70 \%$ of people with lung cancer are still diagnosed at an advanced or metastatic stage and shortened life span often generates high stress for these patients who are considered a challenging population of cancer patients with diseasespecific care needs. A diagnosis of lung cancer is often associated with high co-morbidities which considerably reduce patient's quality of life [12] and many studies reveal that this disease and its treatments have more than a physical impact. Sanders et al. [13] identified the greatest needs of lung cancer patients in the physical and daily living domain, followed by psychological, social and economic needs as well as information and patient care support needs. Lung cancer has a strong impact on patients' families too, who support patients both for the logistic aspects and for the management of symptoms at home, covering psychological and social issues. Several studies demonstrated that caregivers have some difficulties in facing patient-related problems and for this reason, receiving psycho-oncological help and support may improve the quality of life not only for patients but also for caregivers. This result is actually what came to light from the mentioned survey, specifically in Poland and Japan. In this context, it is also crucial to train the doctors in communication and it is very important to be able to build an open relationship between the patient and the healthcare professional, which can influence the effectiveness of the therapy and the psychological health of the patient. 


\section{Information \& communication}

As already stated, lung cancer has a significant impact on both patients and their families and the communication of the diagnosis with a discussion of different treatment options can be overwhelming for patients and their families, so while many of them fail to understand the prognosis and goals of the treatment, most physicians are unaware of these misunderstandings [14].

Different studies have documented patients' and family members' dissatisfaction with the information received and have shown that building a clear and adequate patient-clinician communication is critical in cancer care where the situation adds a potentially greater emotional dimension. The high levels of co-morbidities, the side effects of treatment, the fear, sadness and anxiety associated with having lung cancer are very severe but often lack the required attention by the healthcare team. A comprehensible communication plays a key role in improving patients' knowledge about the prognosis, treatment options and side effect management, in helping patients to better cope with the condition, reducing uncertainty and distress and increasing the adherence to treatments and clinician recommendations.

Bearing in mind that doctor's time is limited and the lack in time being a substantial barrier for effective communication trainings for clinician-patient communication should respond to this fact.

In conclusion, in lung cancer, there is a strong need to overcome many challenges and barriers to ensure that all of the patients may have the same hope and equal chances to fight against this disease regardless of the country of residence. Lung cancer is still considered the most common cancer and in many countries it is the top cause of death. As outlined in this article and confirmed by the results of the survey and the outcome of the interactive session in Wien, lung cancer patients and caregivers still face a number of significant challenges and more has to be done to encourage early diagnosis, to increase public awareness, to provide diagnostic tools and access to safe and effective treatments, to support efficient research and to combat the stigma.

\section{Financial \& competing interests disclosure}

The authors have no relevant affiliations or financial involvement with any organization or entity with a financial interest in or financial conflict with the subject matter or materials discussed in the manuscript. This includes employment, consultancies, honoraria, stock ownership or options, expert testimony, grants or patents received or pending, or royalties.

No writing assistance was utilized in the production of this manuscript.

\section{References}

1 A recent survey across 10 countries highlighted that there is significant lack of understanding about the widespread impact of lung cancer, but also a critical absence of knowledge around the role of genetic changes in the development of the disease. Any One Any Lung Global Survey Results - November 2014 NOVARTIS G-LDK-1102619 www.novartisoncology.com/anyoneanylung

2 Ferlay J, Soerjomataram I, Dikshit R et al. Cancer incidence and mortality worldwide: sources, methods and major patterns in GLOBOCAN 2012. Int. J. Cancer 136, E359-E386 (2015).

3 European Respiratory Society. European Lung White Book. www.erswhitebook.org/files/public/ Chapters/19_lung_cancer.pdf

4 Kauczor HU, Bonomo L, Gaga M et al. ESR/ ERS white paper on lung cancer screening. Eur. Respiratory Journal 46, 29 (2015). http://erj.ersjournals.com/content/

\section{early/2015/04/29/09031936.00033015}

5 Corner J. Is late diagnosis of lung cancer inevitable? Interview study of patients' recollections of symptoms before diagnosis. Thorax 60, 314-319 (2005).

6 Global Perception of Lung Cancer: An Ipsos MORI report for the Global Lung Cancer Coalition Based on WHO data (2005) on prevalence of tobacco used by country. www.who.int/mediacentre/factsheets/fs297/ en/index.html

7 Aggarwal A, Lewison G, Idir S et al. The State of Lung Cancer Research: A Global Analysis. J. Thoracic Oncol. http://dx.doi.org/10.1016/j. jtho.2016.03.010

8 Kauczor $\mathrm{H}$ et al. 4629 ESR/ERS white paper on lung cancer screening. Eur. Respir. J. (2015). http://erj.ersjournals.com/content/ early/2015/04/29/09031936.00033015

9 Worley S. Lung cancer research is taking on new challenges: knowledge of tumors' molecular diversity is opening new pathways to treatment. PT 39(10), 698-714 (2014).
10 Anant M, Guleria R, Pathak AK et al. Quality of life measures in lung cancer. Indian J. Cancer 15, 125-132 (2005).

11 Committee on Psychosocial Services to Cancer Patients/Families in a Community Setting. Cancer Care for the Whole Patient: Meeting Psychosocial Health Needs. Adler NE, Page AEK (Eds).

12 Quirt CF, Mackillop WJ, Ginsburg AD et al. Do doctors know when their patients don't? A survey of doctor-patient communication in lung cancer. Lung Cancer 18, 1-20 (1997).

13 Sanders SL, Bantum EO, Owen JE, Thornton AA Stanton AL. Supportive care needs in patients with lung cancer. Psycho-Oncology. (2010).

14 Quirt CF, Mackillop WJ, Ginsburg AD, et al. Do doctors know when their patients don't? A survey of doctor-patient communication in lung cancer. Lung Cancer 18, 1-20 (1997). 